\title{
Poor sleep quality is negatively associated with low cognitive performance in general population independent of self-reported sleep disordered breathing
}

\author{
Zhongrong Wang 1,2,3,4,5 ${ }^{\dagger}$, Mulalibieke Heizhati ${ }^{1,2,3,4,5 \dagger}{ }^{\text {, Lin Wang }}{ }^{1,2,3,4,5}$, Mei Li 1,2,3,4,5, Zhikang Yang 1,2,3,4,5, \\ Mengyue Lin 1,2,3,4,5, Reyila Abudereyimu 1,2,3,4,5, Jing Hong 1,2,3,4,5, Wenbo Yang 1,2,3,4,5, Ling Yao 1,2,3,4,5, \\ Shasha Liu', $1,3,4,5$, Junli Hu ${ }^{1,2,3,4,5}$ and Nanfang Li ${ }^{1,2,3,4,5^{*}}$
}

\begin{abstract}
Background: Sleep disordered breathing (SDB) plays a significant role in both sleep quality and cognition and whether it has an impact on the relationship between above two factors remains to be clear. The study aimed to explore the association between sleep quality and cognitive performance in general population by considering influence of sleep disordered breathing (SDB).

Methods: In this cross-sectional study, we enrolled subjects aged $\geq 18$ years using a multi-stage random sampling method. Cognitive status was assessed using Mini Mental State Examination (MMSE) questionnaire, sleep quality using Pittsburgh Sleep Quality Index (PSQI) and SDB was assessed using No-SAS scale, respectively. Multi-variable logistic regression was applied to examine the association of sleep quality and cognitive performance. Subgroup analyses were performed in different age groups, and in those with and without SDB.

Results: Finally, 30,872 participants aged $47.5 \pm 13.8$ years with $53.5 \%$ women were enrolled, of whom $32.4 \%$ had poor sleep quality and $18.6 \%$ had low cognitive performance. Compared with good sleepers, subjects with poor sleep quality exhibited significantly higher presence of low cognitive performance ( $23.7 \%$ vs $16.2 \%, P<0.001)$. Poor sleepers revealed 1.26 (95\%Cl: 1.16,1.36), 1.26 (1.08,1.46) and $1.25(1.14,1.37)$ fold odds for low cognitive performance in general population and in subjects with and without self-reported SDB respectively. Stratified by age and SDB, the association was observed in young and middle-aged group without SDB $(\mathrm{OR}=1.44,95 \% \mathrm{Cl}: 1.30,1.59)$ and in the elderly group with SDB (OR=1.30, 95\%Cl: 1.07,1.58).
\end{abstract}

Conclusions: Sleep quality is in a negative association with cognitive performance in general population independent of SDB, implying improvement of sleep disturbances is a potential objective of intervention strategies for cognitive protection at population level.

\footnotetext{
*Correspondence: Inanfang2016@sina.com

'Zhongrong Wang and Mulalibieke Heizhati contributed equally to this work and share co-first authorship.

${ }^{1}$ Hypertension Center of People's Hospital of Xinjiang Uygur

Autonomous Region, Xinjiang Hypertension Institute National Health

Committee Key Laboratory of Hypertension Clinical Research, No. 91

Tianchi Road Urumqi, Xinjiang 830001, China

Full list of author information is available at the end of the article
}

(c) The Author(s) 2022. Open Access This article is licensed under a Creative Commons Attribution 4.0 International License, which permits use, sharing, adaptation, distribution and reproduction in any medium or format, as long as you give appropriate credit to the original author(s) and the source, provide a link to the Creative Commons licence, and indicate if changes were made. The images or other third party material in this article are included in the article's Creative Commons licence, unless indicated otherwise in a credit line to the material. If material is not included in the article's Creative Commons licence and your intended use is not permitted by statutory regulation or exceeds the permitted use, you will need to obtain permission directly from the copyright holder. To view a copy of this licence, visit http://creativecommons.org/licenses/by/4.0/. The Creative Commons Public Domain Dedication waiver (http://creativeco mmons.org/publicdomain/zero/1.0/) applies to the data made available in this article, unless otherwise stated in a credit line to the data. 
Keywords: Sleep quality, Sleep disordered breathing, Low cognitive performance

\section{Introduction}

Cognitive impairment including dementia and Alzheimer's disease (AD) has become one of the most serious public health problems worldwide [1, 2]. About 35.6 million people lived with dementia in 2010, with the number expected to double every 20 years [3]. Currently, there are no effective treatment options for the disease, leaving control of risk factors and prevention at pre-clinical stage as the effective way to delay disease onset [4].

Mild cognitive impairment (MCI), or low cognitive performance, is considered a transition stage between healthy cognitive aging and dementia, and can be identified years before dementia onset [5]. Nearly 2.4-42\% population aged $\geq 60$ suffer from MCI, based on studies from a number of countries using different screening and diagnostic cutoffs $[6,7]$. Conversion rate from amnestic form of MCI to Alzheimer's disease is estimated to be $10-15 \%$ per year [8].

Existing evidence suggests that poor sleep quality, per se a public health issue with a prevalence of $6 \%-94 \%$ in adults [9] be a risk factor for cognitive decline [10]. In Heinz Nixdorf Recall study, one of few existing relevant longitudinal studies, poor sleep quality, assessed using Pittsburgh Sleep Quality Index (PSQI), is associated with incident $\mathrm{MCI}$ in participants aged $\geq 50$ years during mean follow-up 5.2 years [10]. In addition, in a meta analysis for cross-sectional and cohort studies of community dwellers, individuals with sleep problems including poor sleep quality show a pooled 1.68 times higher risk for combined outcome of cognitive impairment [11]. Furthermore, animal models indicate protective effects of better sleep quality on cognition by removal of neurotoxic waste products from central nervous system [12]. Nonetheless, results from studies are not always consistent, with some reporting null association between the two $[13,14]$. The heterogeneity of results between these studies could be due to a number of methodological differences, including age [13-15] and sex [14] of participants, population culture or ethnicity $[13,15,16]$, cognitive assessments or sleep measures [16], and statistical adjustments made for various potential confounders [13-15].

Importantly, effects of sleep disordered breathing (SDB) on the association of the two were not considered or not evaluated systematically in previous studies [1315]. SDB is an umbrella term, with a prevalence of $24.0 \%$ 83.8\% [17] and with OSA as the most frequent type [18]. OSA is a well-established risk factor for cognitive decline [19] and shows a strong association with AD [20], which could be explained by the fact that hypoxia plays a vital role and may contribute to increases in $A \beta$ production, thereby facilitating AD pathogenesis [19]. Furthermore, Dlugaj et al. reported in small sample individuals aged 45-75 years that poor sleep quality is associated with low cognitive performance independent of apnea hypopnea index, a parameter of OSA severity [16]. Meanwhile, emerging evidence shows that sleep quality improvements can be achieved using cost-effective interventions and are beneficial in disease prevention [21]. Therefore, considering the high prevalence of SDB in general population, the well documented associations between SDB with sleep quality and cognitive performance, it might be of important significance to evaluate whether the relationship between sleep quality and low cognitive performance is independent of SDB, which may help public health interventions to formulate sleep quality improvement programs for cognitive protection at population level.

To our knowledge, there is few large-scale populationbased epidemiological surveys to assess the association due to difficulty in assessing SDB in population-based studies. Current guidelines recommend polysomnography (PSG) as the gold standard for SDB assessment, which is an expensive time-consuming, labor-intensive, and technique dependent equipment, and inconvenient to be utilized in large scale population-based studies [22]. Nonetheless, NOSAS scale is a simple, efficient, and easy method for screening of self-reported SDB [23] and validated in some of Chinese population [24, 25] with a high sensitivity and specificity.

Therefore, the main objective of current article is to examine the association of overall sleep quality and low cognitive performance, by considering the influence of self-reported SDB in a large sample, representing the general adult population.

\section{Methods}

Site

Emin County, Northern Xinjiang China, is home to multi-ethnic population of over 160,000, living in urban, agricultural and stock-raising settings with higher prevalence of risk factors for cardiovascular disease with poor management $[26,27]$.

\section{Study population}

In this cross-sectional study, we used multi-stage stratified sampling method to enroll study population aged $\geq 18$ years. At the $1^{\text {st }}$ stage, Emin county was divided 
into urban, agricultural and stock raising settings as in our previous study [27]. At the $2^{\text {nd }}$ stage, two corresponding streets, villages or groups were selected using sample random sampling (SRS) method. At the $3^{\text {rd }}$ stage, subjects aged $\geq 18$ years were selected using SRS method from the residence data. Inclusion criteria encompassed: 1. local inhabitants aged $\geq 18$ years and residing at current address for $\geq 6$ months; 2 . agreement to participate and to sign an informed consent form. Exclusion criteria included confirmed dementia, confirmed $A D$, malignant tumors including those in central nervous system by computerized tomography and magnetic resonance imaging, encephalitis or meningitis, injury, trauma and or operations in central nervous system; those who are unable to co-operate due to mental reasons or others, and sever diseases on respiratory and or digestive system and night workers. To have higher response rate, survey was conducted together with annual health check up program between March to November 2019.

\section{Data collection}

Each participant completed a questionnaire including PSQI, Mini Mental State Examination (MMSE), Global Physical Activity Questionnaire (GPAQ), Zung's Self Rating Anxiety and Depression Scale (SAS, SDS) questionnaires. Data were also collected on participants' demographic characteristics (age, gender, ethnicity), socioeconomic status (occupation, education status), lifestyles (cigarette and alcohol consumption) and medical histories (such as hypertension).

Trained staff measured body height, weight, neck circumference (NC), waist circumference (WC), and blood pressure (BP) of each participant using standardized protocol. Before BP measurement, each participant was asked to avoid drinking alcohol, smoking cigarettes and drinking coffee or tea. Each participant's BP records were obtained using with OMRON HBP-1300 Professional Portable Blood Pressure Monitor (OMRON, Kyoto, Japan) three times on the right arm positioned at heart level with participant sitting at rest for five minutes, with 30 seconds between each measurement with an observer present. Average of three readings was used for analysis. Overnight fasting blood samples were obtained for measuring serum fasting blood glucose (FBG), lipid profiles and creatinine with clinical standard procedures at local hospitals.

\section{Assessment of cognitive status}

Trained investigators evaluated cognitive status with MMSE [28], with a total score ranging from 0-30. Low cognitive performance is defined as MMSE score $<17,<20$ and $<24$ for subjects with no formal education, 1-6 years of and with $\geq 7$ years of education respectively [29].

\section{Assessment of sleep quality}

Sleep quality was assessed with PSQI [30], the most used self-report instrument for assessing sleep quality of preceding 4 weeks. It was consisted with subjective sleep quality, sleep latency, sleep duration, sleep efficiency, sleep disturbances, use of sleep medication, and daytime dysfunction. A global score ranges from 0 to 21 and score $\geq 6$ indicates poor sleep quality [31].

\section{Assessment of SDB}

NoSAS score was used to assess self-reported SDB, score of which ranges from 0 to 17. self-reported SDB was defined with a NoSAS score $\geq 8$ [23].

\section{Definitions of co-variables}

Hypertension is defined as systolic $\mathrm{BP} \geq 140 \mathrm{mmHg}$ and/ or diastolic $\mathrm{BP} \geq 90 \mathrm{mmHg}$ and/or receiving anti-hypertensive medication within previous 2 weeks [32]. Diabetes mellitus is defined as $\mathrm{FBG} \geq 7.0 \mathrm{mmol} / \mathrm{L}$, and/or self-reported previously diagnosed by physicians and/or intake of hypoglycemic agents within past 2 weeks [33]. Dyslipidemia is defined as total cholesterol $\geq 6.2 \mathrm{mmol} / \mathrm{L}$ and/or triglyceride $\geq 2.3 \mathrm{mmol} / \mathrm{L}$ and/or high density lipoprotein cholesterol $<1.0 \mathrm{mmol} / \mathrm{L}$ and/or low density lipoprotein cholesterol $\geq 4.2 \mathrm{mmol} / \mathrm{L}$ and/or having received treatment during past 2 weeks [34]. Coronary heart disease (CHD) and stroke were self-reported by the participants. Anemia was defined based on hemoglobin $<12 \mathrm{~g} / \mathrm{dL}$ for men and $<11 \mathrm{~g} / \mathrm{dL}$ for women [35]. Standard SAS and SDS score of 45 and 50 is set as cutoff for presence of anxiety and depression status respectively [36, 37]. Physical activity levels were grouped into high ( $\geq 3000$ MET-minutes per week from any combination of walking, moderate-or vigorous-intensity activities or $\geq 1500$ MET-minutes per week from vigorous intensity activity), moderate (not meeting the criteria for the high category, but achieving $\geq 600$ MET-minutes per week) and low (not meeting any of the above criteria) categories [38]. Alcohol intake is defined as consuming alcoholic beverage at least once per week in the past month. Cigarette consumption is defined as participants who have smoked at least 20 packets of cigarettes in their lifetime and currently smoke cigarettes and non-smokers as participants who never smoked or smoked $<20$ packets of cigarettes in their entire lifetime.

\section{Statistical analysis}

Current study is a post-hoc analysis of the whole data collected in Emin in 2019. Students' t-test was used to assess between-group differences in continuous variables if 
normally distributed; otherwise, nonparametric (MannWhitney $\mathrm{U}$ ) test was applied. $\mathrm{X}^{2}$-test was used to assess between-group differences of categorical variables. $\mathrm{P}$ for trend was calculated by Kruskal-Wallis $\mathrm{H}$ test and $\mathrm{X}^{2}$ trend test for ordinal variables. Multiple logistic regression was used to estimate unadjusted and adjusted odds ratios (ORs) and 95\% confidence intervals (95\% CIs) for the association between poor sleep quality and low cognitive performance. Tolerance and variance inflation factor (VIF) were examined to identify multicollinearity and multicollinearity is a concern if VIF is $>10$ and the tolerance is $<0.10$ [39]. Before creating regression models, independent variables significantly relevant to low cognitive performance were selected using bi-variate analysis. Model a was adjusted for age and gender. Model b was adjusted for model a plus ethnicity, marital status, education attainment status, occupation, living setting, cigarette and alcohol consumption, BMI, physical activity level, estimated glomerular filtration rate (eGFR), anemia, anxiety and depression status, hypertension, dyslipidemia, diabetes mellitus, CHD and stroke. Model c was additionally adjusted for self-reported SDB. Age and or self-reported SDB was removed from the model when it was the stratification variable. Results were considered statistically significant if two-tailed $p$ value was less than 0.05. All statistical analyses were performed with SPSS statistical software, version 24.0 (Chicago, IL, USA).

\section{Results}

\section{Participants characteristics at baseline}

Finally, a total of 30,872 participants with complete MMSE and PSQI data were comprised analytical sample, with an average age of 47.5 and $46.5 \%$ of men, of whom $32.4 \%$ suffered from poor sleep. Participants with poor sleep quality were older, having higher BMI, more likely to be women and take manual labor and less likely to smoke and drink, compared those with good sleep quality. Individuals with chronic diseases such as hypertension, dyslipidemia, diabetes mellitus, CHD, stroke, depression, anxiety and self-reported SDB were more likely to have poor sleep quality (Table 1).

\section{MMSE scores and presence of low cognitive performance}

Median MMSE score was 26 and overall prevalence of low cognitive performance was $18.6 \%$ in total study participants. MMSE score was significantly lower in poor sleep quality group ( 25.0 vs $27.0, P<0.001)$ and accordingly prevalence of low cognitive performance was significantly higher $(23.7 \%$ vs $16.2 \%, P<0.001)$, compared with the good sleep quality group. Similar results were observed in different age and self-reported SDB subgroups (Fig. 1).

\section{Association between sleep quality and low cognitive} performance (Table 2)

Compared to the reference group of cognitively impaired individuals, odds for presence of low cognitive performance in poor sleep quality group was $1.26(95 \% \mathrm{CI}$ : $1.16,1.36)$ in total population, 1.26 (95\% CI: 1.08, 1.46) in individuals suffering from self-reported SDB and 1.25 (95\%CI: 1.14, 1.37) in those free of self-reported SDB after adjusting relevant confounders. When stratified by age, poor sleep quality was also associated with presence of low cognitive performance in participants aged $\leq 59$ $(O R=1.43,95 \% \mathrm{CI}: 1.30,1.56)$ and those aged $\geq 60$ $(O R=1.17,95 \% C I: 1.02,1.34)$. Nevertheless, the association only remained in individuals aged $\leq 59$ without selfreported SDB $(O R=1.44,95 \% \mathrm{CI}: 1.30,1.59)$ and those aged $\geq 60$ with self-reported SDB $(O R=1.30,95 \% \mathrm{CI}$ : $1.07,1.58)$ in cross stratification analysis.

\section{Discussion}

Current investigation is one of few large-scale population-based studies to explore the relationship between sleep quality and cognitive performance by considering SDB as a confounder. Main results encompass: 1) As sleep quality deteriorates, MMSE total score declines and presence of low cognitive performance ascends progressively. 2) Sleep quality is negatively associated with presence of low cognitive performance in general population independent of self-reported SDB. 3) When stratified by age, the association is confined to individuals aged $\leq 59$ years without self-reported SDB and those aged $\geq 60$ years with self-reported SDB.

In support of current results, population-based prospective cohort studies in community dwellers report that poor sleepers harbour higher risk for developing low cognitive performance than their counterparts [10]. In addition, in a meta analysis of observational studies including cross-sectional and cohort studies of community dwellers aged $>60$ years, individuals with sleep problems show higher risk for the combined outcome of cognitive impairment than those with good sleep [11]. However, effects of SDB on the association of sleep quality and cognitive performance in population-based studies have remained less investigated.

One of the differences of the current study with previous population-based ones is the inclusion of selfreported SDB as a variable. And we observed that sleep quality is negatively associated with low cognitive performance independent of SDB in total participants and in individuals aged $\leq 59$ years, but dependent on SDB in those aged $\geq 60$ years. In line with current results, Dlugaj et al. reported in a small sample individuals aged 45 to 75 years that poor sleep quality is associated with low 
Table 1 Characteristics of study population by sleep quality

\begin{tabular}{|c|c|c|c|c|}
\hline & Total $(n=30,872)$ & Good $(n=20,866)$ & Poor $(n=10,006)$ & $\mathrm{t}, \mathrm{X}^{2}, \mathrm{Z} / \mathrm{P}$ \\
\hline Gender (men,n,\%) & $14,347(46.5)$ & $10,728(51.4)$ & $3619(36.2)$ & $631.894 /<0.001$ \\
\hline Age (Mean $\pm S D$, years) & $47.5 \pm 13.8$ & $45.4 \pm 13.4$ & $51.8 \pm 13.5$ & $39.132 /<0.001$ \\
\hline Age group $(n, \%) \leq 44$ & $12,570(40.7)$ & $9797(47.0)$ & $2773(27.7)$ & $1323.068 /<0.001$ \\
\hline $45-59$ & $12,213(39.6)$ & $7891(37.8)$ & $4322(43.2)$ & \\
\hline$\geq 60$ & $6089(19.7)$ & $3178(15.2)$ & $2911(29.1)$ & \\
\hline Ethnicity $(n, \%)$, Han & $15,318(49.7)$ & $10,051(48.2)$ & $5267(52.7)$ & $89.052 /<0.001$ \\
\hline Kazakh & $10,236(33.2)$ & $7282(34.9)$ & $2954(29.6)$ & \\
\hline Others & $5293(17.2)$ & $3520(16.9)$ & $1773(17.7)$ & \\
\hline Marital status $(n, \%)$ unmarried & $2431(7.9)$ & $1927(9.3)$ & $504(5.0)$ & $441.202 /<0.001$ \\
\hline married & $25,571(83.0)$ & $17,414(83.7)$ & $8157(81.7)$ & \\
\hline divorced/widowed & $2790(9.1)$ & $1467(7.1)$ & $1323(13.3)$ & \\
\hline Education $(n, \%), \leq$ primary & $9858(32.0)$ & $5999(28.8)$ & $3859(38.6)$ & $305.939 /<0.001$ \\
\hline Junior high & $11,716(38.0)$ & $8372(40.2)$ & $3344(33.5)$ & \\
\hline$\geq$ Senior high & $9247(30.0)$ & $6460(31.0)$ & $2787(27.9)$ & \\
\hline Region $(n, \%)$, urban & $11,058(36.2)$ & $7023(34.0)$ & $4035(40.8)$ & $143.364 /<0.001$ \\
\hline stock-raising & $3669(12.0)$ & $2652(12.8)$ & $1017(10.3)$ & \\
\hline agricultural & $15,823(51.8)$ & $10,974(53.1)$ & $4849(49.0)$ & \\
\hline Occupation $(n, \%)$, mental labour & $8898(30.2)$ & $6142(30.9)$ & $2756(28.8)$ & $14.186 /<0.001$ \\
\hline physical labor & $20,519(69.8)$ & $13,704(69.1)$ & $6815(71.2)$ & \\
\hline Current smokers $(n, \%)$ & $7748(26.3)$ & $5766(28.8)$ & $1982(20.9)$ & $207.420 /<0.001$ \\
\hline Current drinkers $(n, \%)$ & $2807(9.2)$ & $2010(9.7)$ & $797(8.0)$ & $22.728 /<0.001$ \\
\hline Body mass index (Mean $\pm S D, k g / \mathrm{m}^{2}$ ) & $25.8 \pm 4.3$ & $25.7 \pm 4.2$ & $26.1 \pm 4.4$ & $8.059 /<0.001$ \\
\hline Body mass index $\geq 25(n, \%)$ & $16,776(54.9)$ & $11,101(53.7)$ & $5675(57.2)$ & $33.796 /<0.001$ \\
\hline Systolic pressure (Median,quartile) & $123(111,139)$ & $122(111,137)$ & $126(114,141)$ & $14.317 /<0.001$ \\
\hline Diastolic pressure (Median,quartile) & $79(70,88)$ & $79(70,87)$ & $80(71,88)$ & $5.801 /<0.001$ \\
\hline Hypertension (n,\%) & $11,200(36.3)$ & $6816(32.7)$ & $4384(43.8)$ & $363.582 /<0.001$ \\
\hline Dyslipidemia $(n, \%)$ & $9114(29.6)$ & $5414(26.0)$ & $3700(37.1)$ & $395.841 /<0.001$ \\
\hline Diabetes mellitus $(n, \%)$ & $3147(10.2)$ & $1861(8.9)$ & $1286(12.9)$ & $114.166 /<0.001$ \\
\hline Coronary heart disease $(n, \%)$ & $422(1.4)$ & $165(0.8)$ & $257(2.6)$ & $158.695 /<0.001$ \\
\hline Self-reported stroke $(n, \%)$ & $1060(3.5)$ & $465(2.2)$ & $595(6.0)$ & $285.744 /<0.001$ \\
\hline Depression $(n, \%)$ & $1202(3.9)$ & $332(1.6)$ & $870(8.8)$ & $916.267 /<0.001$ \\
\hline Anxiety $(n, \%)$ & $2170(7.1)$ & $556(2.7)$ & $1614(16.2)$ & $1885.621 /<0.001$ \\
\hline NoSAS score (Median,quartile) & $5(2,7)$ & $5(2,7)$ & $5(3,8)$ & $15.820 /<0.001$ \\
\hline Sleep disordered breathing $(n, \%)$ & $7265(23.5)$ & $4547(21.8)$ & $2718(27.2)$ & $108.469 /<0.001$ \\
\hline Physical activity $(n, \%)$, low & $4213(13.9)$ & $2792(13.7)$ & $1421(14.5)$ & $8.377 / 0.015$ \\
\hline moderate & $8220(27.2)$ & $5500(26.9)$ & $2720(27.7)$ & \\
\hline high & $17,823(58.9)$ & $12,160(59.5)$ & $5663(57.8)$ & \\
\hline Serum creatinine (Median,quartile) & $69.2(58.9,81.0)$ & $69.5(59.4,81.2)$ & $68.2(57.8,80.8)$ & $5.963 /<0.001$ \\
\hline eGFR (Median,quartile) & $99.9(83.8,111.3)$ & $102.1(86.2,113.1)$ & $95.6(78.8,107.1)$ & $23.266 /<0.001$ \\
\hline Total cholesterol (Median,quartile) & $4.7(4.0,5.5)$ & $4.7(4.0,5.5)$ & $4.8(4.1,5.6)$ & $11.683 /<0.001$ \\
\hline Triglyceride (Median,quartile) & $1.2(0.9,1.8)$ & $1.2(0.8,1.7)$ & $1.3(0.9,1.8)$ & $6.904 /<0.001$ \\
\hline Fasting blood glucose (Median,quartile) & $5.3(4.8,5.9)$ & $5.3(4.8,5.8)$ & $5.4(4.9,6.0)$ & $10.022 /<0.001$ \\
\hline
\end{tabular}

eGFR estimated glomerular filtration rate

cognitive performance independent of apnea hypopnea index, an indicator of OSA severity assessed objectively using PSG [16]. Furthermore, as previously reported, whether treatment of SDB with continuous positive airway pressure (CPAP) reverses cognition impairment remains unknown [40], whereas CPAP improves sleep quality [41]. Importantly, simple interventions for improving sleep quality such as sleep hygiene and chronobiotics can also delay the progress of cognitive impairment [21]. However, based on limited evidence, it 

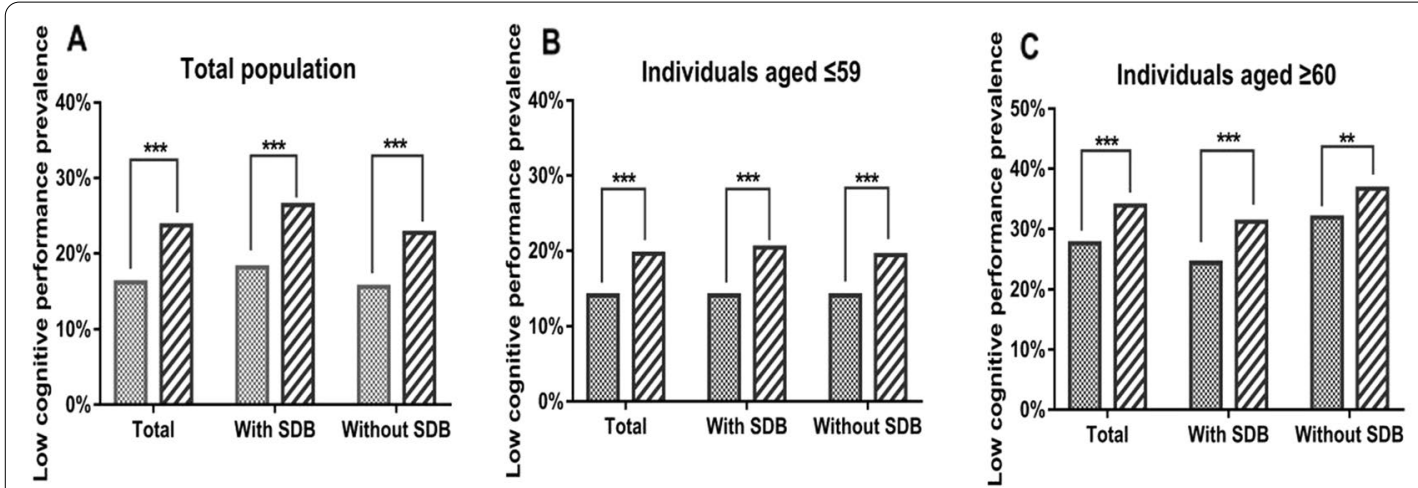

Good sleep quality

Poor sleep quality

Fig. 1 Prevalence of low cognitive performance by sleep quality in total population (A), individuals aged $\leq 59$ (B) and $\geq 60$ years $(\mathbf{C})$, with and without SDB (*** and ${ }^{* *}$ indicated statistical significance $P \leq 0.001$ and $\leq 0.005$, respectively.)

Table 2 Associations between sleep quality and $\mathrm{MCl}$ by logistic regression models in total population and age subgroups by presence of $S D B$

\begin{tabular}{|c|c|c|c|c|}
\hline & Crude OR $(95 \% \mathrm{Cl}), P$ & Adjusted $^{\mathrm{a}}$ OR(95\%Cl), $P$ & Adjusted $^{\mathrm{b}}$ OR(95\%Cl), $P$ & Adjusted $^{\mathrm{C}} \mathrm{OR}(95 \% \mathrm{Cl}), P$ \\
\hline \multicolumn{5}{|l|}{ Total population } \\
\hline Good sleep quality & Ref & Ref & Ref & Ref \\
\hline Poor sleep quality & $1.61(1.52,1.71),<0.001$ & $1.24(1.17,1.32),<0.001$ & $1.26(1.16,1.36),<0.001$ & $1.26(1.16,1.36),<0.001$ \\
\hline \multicolumn{5}{|l|}{ Individuals with SDB } \\
\hline Good sleep quality & Ref & Ref & Ref & \\
\hline Poor sleep quality & $1.61(1.44,1.80),<0.001$ & $1.24(1.10,1.40)_{,}<0.001$ & $1.26(1.08,1.46), 0.003$ & \\
\hline \multicolumn{5}{|c|}{ Individuals without SDB } \\
\hline Good sleep quality & Ref & Ref & Ref & \\
\hline Poor sleep quality & $1.59(1.48,1.70),<0.001$ & $1.24(1.16,1.34),<0.001$ & $1.25(1.14,1.37),<0.001$ & \\
\hline \multicolumn{5}{|c|}{ Population aged $\leq 59$ years } \\
\hline Good sleep quality & Ref & Ref & Ref & Ref \\
\hline Poor sleep quality & $1.48(1.38,1.59),<0.001$ & $1.42(1.32,1.53),<0.001$ & $1.43(1.30,1.57),<0.001$ & $1.43(1.30,1.56),<0.001$ \\
\hline \multicolumn{5}{|l|}{ Individuals with SDB } \\
\hline Good sleep quality & Ref & Ref & Ref & \\
\hline Poor sleep quality & $1.56(1.31,1.86),<0.001$ & $1.29(1.08,1.56), 0.006$ & $1.26(0.99,1.59), 0.056$ & \\
\hline \multicolumn{5}{|c|}{ Individuals without SDB } \\
\hline Good sleep quality & Ref & Ref & Ref & \\
\hline Poor sleep quality & $1.46(1.35,1.58),<0.001$ & $1.42(1.31,1.54),<0.001$ & $1.44(1.30,1.59)_{,}<0.001$ & \\
\hline \multicolumn{5}{|c|}{ Population aged $\geq 60$ years } \\
\hline Good sleep quality & Ref & Ref & Ref & Ref \\
\hline Poor sleep quality & $1.34(1.21,1.50),<0.001$ & $1.24(1.11,1.39)_{,}<0.001$ & $1.16(1.01,1.33), 0.033$ & $1.17(1.02,1.34), 0.029$ \\
\hline \multicolumn{5}{|l|}{ Individuals with SDB } \\
\hline Good sleep quality & Ref & Ref & Ref & \\
\hline Poor sleep quality & $1.41(1.21,1.64),<0.001$ & $1.30(1.11,1.52), 0.001$ & $1.30(1.07,1.58), 0.008$ & \\
\hline \multicolumn{5}{|c|}{ Individuals without SDB } \\
\hline Good sleep quality & Ref & Ref & Ref & \\
\hline Poor sleep quality & $1.24(1.06,1.45), 0.008$ & $1.19(1.02,1.40), 0.031$ & $1.04(0.85,1.27), 0.705$ & \\
\hline
\end{tabular}

${ }^{\mathrm{a}}$ adjusted for age, gender; ${ }^{\mathrm{b}}$ model a plus ethnicity, marriage status, education status, occupation, region, cigarette consumption, alcohol intake, body mass index, physical activity level, hypertension, diabetes mellitus, dyslipidemia, stroke, coronary heart disease, estimated glomerular filtration rate, anemia, depression and anxiety status; ${ }^{C}$ model b plus sleep disordered breathing. Removing age when it was the stratification variable. OR, odds ratio $C l$, confidence interval $S D B$, sleep disordered breathing 
is difficult to define that sleep quality and self-reported SDB are independently related with cognitive decline. Therefore, future studies are needed to explore the nature of relationships between different parameters of sleep and variables of cognition.

Inconsistent association of sleep quality, self-reported SDB and cognitive function in the elderly and young and middle-aged population has also been observed in a previous study [42]. Possible reasons for this phenomenon may include the following. Prevalence of both poor sleep quality and SDB increases with age and is highly prevalent in the elderly [43], as high as $62.4 \%$ [44] and $80 \%$ [45] respectively. This may blur the real association or effects of SDB on the association may weigh poor sleep quality. On the other hand, subjective assessment of changes in sleep quality using questionnaire like PSQI, may not be sensitive in the elderly. As an example, in a populationbased study, generic complaints of sleep disturbances declined across the life span, with the highest in the aged 18-24 years, peaked again in the aged 45-59 years and the lowest in the elderly [46]. However, further studies are needed in this specific population group.

Several strengths merit this study as followings: First, this is one of the few large-scale population-based epidemiological studies to explore the association between sleep quality and cognitive performance involving SDB. Second, multiple confounders have been adjusted in the study such as self-reported SDB, depression, anxiety, eGFR, physical activity and so on, which are previously proven to be associated with low cognitive performance $[47,48]$, making the current results more reliable ones. Third, with a multi-stage random sampling method, participants in this survey are in a wide age range of men and women from general population and results can be applied to general population. However, a few limitations should also be considered, while explaining the data. Cross-sectional design makes it difficult to define causality and directionality of the association between sleep quality and low cognitive performance. Nonetheless, current results are in line with previous population-based cohort studies, in which baseline poor sleep quality is associated with cognitive decline during follow up [10]. Therefore, it may indicate current results are reliable. And we further extended previous results to those with and without self-reported SDB by considering the age. Second, we assessed subjective sleep quality using PSQI. Although it entails some subjectivity, PSQI is a more convenient method to identify poor sleep quality in practice than the objective but prohibitively expensive methods such as PSG [22]. Previous studies revealed that PSQI global score has moderate associations with some objective sleep quality indexes including PSG sleep maintenance, sleep efficiency, and microarousal index [31]. Considering the high internal consistency $(\alpha=0.83)$, test- retest reliability $(r=0.85)$ [30], and moderate structural validity identifying subjects with poor sleep quality in both clinical and non-clinical populations [31], PSQI is a popular, reliable and valid instrument. Third, effectiveness of PSQI for evaluating sleep quality in population with low cognitive performance remains to be evaluated, which may have some bias on current results. Importantly, interventions for improving sleep quality have been shown to delay progress of cognitive impairment [21]. Fourth, one time assessment of cognitive function by MMSE is also a limitation. Combined with other tests of cognitive function (such as Montreal cognitive assessment) will help to make more augmented association with sleep quality. However, MMSE is a worldwide used measurement for cognitive screening [28] with the same cut-offs demonstrating high validity [49] and even suitable in younger population [50].

In conclusion, sleep quality is in a negative association with prevalent low cognitive performance in general population independent of self-reported SDB, implying improvement of sleep disturbances is a potential objective of intervention strategies for cognitive protection at population level.

\section{Acknowledgements \\ We gratefully acknowledge all of the study participants during the study.}

\section{Authors' contributions}

Study concepts/study design were performed by NFL, MLLBKHZT and LW. Data acquisition was performed by ZRW, MLLBKHZT, LW, ML, ZKY, MYL, RYLABDRYM, JH, WBY, SSL, LY, JLH and data analysis/interpretation was performed by ZRW. Manuscript drafting or manuscript revision for important intellectual content was performed by ZRW, MLLBKHZT and NFL. All authors contributed to writing-review and editing. All authors have read and agreed to the published version of the manuscript.

\section{Funding}

This study was supported by Key Research And Development Projects of Xinjiang Uygur Autonomous Region of China (Grant Number: 2017B03015) and Department of Science and Technology of Xinjiang Uygur Autonomous Region of China (Grant number: 2019D01C147).

Availability of data and materials

Data is available from corresponding author upon reasonable request.

\section{Declarations}

\section{Ethics approval and consent to participate}

This study was conducted according to the guidelines laid down in the Declaration of Helsinki and all procedures involving research study participants were approved by The Independent Ethics Committee of People's Hospital of Xinjiang Uygur Autonomous Region. Signed informed consent was obtained from all of the eligible participants.

Consent for publication

Not applicable

Competing interests

The authors declare no conflict of interest. 


\begin{abstract}
Author details
${ }^{1}$ Hypertension Center of People's Hospital of Xinjiang Uygur Autonomous Region, Xinjiang Hypertension Institute National Health Committee Key Laboratory of Hypertension Clinical Research, No. 91, Tianchi Road Urumqi, Xinjiang 830001 , China. ${ }^{2}$ Xinjiang Hypertension Institute, Xinjiang, China. ${ }^{3}$ National Health Committee Key Laboratory of Hypertension Clinical Research, Xinjiang, China. ${ }^{4}$ Key Laboratory of Xinjiang Uygur Autonomous Region "Hypertension Research Laboratory, Xinjiang, China. ${ }^{5}$ Xinjiang Clinical Medical Research Center for Hypertension (Cardio-Cerebrovascular) Diseases, Xinjiang, China.
\end{abstract}

Received: 20 April 2021 Accepted: 9 December 2021

Published online: 03 January 2022

\section{References}

1. Alzheimer's Association. Alzheimer's disease facts and figures. Alzheimers Dement. 2019:15:321-87.

2. Prince M, Comas-Herrera A, Knapp M, et al. World alzheimer report 2016 -improving healthcare for people living with dementia: coverage, quality and costs now and in the future. London: Alzheimer's Disease International; 2016. p. 1-140.

3. Sosa-Ortiz AL, Acosta-Castillo I, Prince MJ. Epidemiology of dementias and Alzheimer's disease. Arch Med Res. 2012:43:600-8.

4. Cummings JL, Morstorf T, Zhong K. Alzheimer's disease drug-development pipeline: few candidates, frequent failures. Alzheimers Res Ther. 2014;6:37.

5. DeCarli C. Mild cognitive impairment: prevalence, prognosis, aetiology, and treatment. Lancet Neurol. 2003;2:15-21.

6. Xue J, et al. The Prevalence of Mild Cognitive Impairment in China: A Systematic Review. Aging Dis. 2018;9:706-15.

7. Ward A, Arrighi HM, Michels S, Cedarbaum JM. Mild cognitive impairment: disparity of incidence and prevalence estimates. Alzheimers Dement. 2012;8:14-21.

8. Janoutová J, Šerý O, Hosák L, Janout V. Is Mild Cognitive Impairment a Precursor of Alzheimer's Disease? Short Review. Cent Eur J Public Health. 2015;23:365-7.

9. Simonelli G, Marshall NS, Grillakis A, Miller CB, Hoyos CM, Glozier N. Sleep health epidemiology in low and middle-income countries: a systematic review and meta-analysis of the prevalence of poor sleep quality and sleep duration. Sleep Health. 2018;4:239-50.

10. Brachem C, Winkler A, Tebrügge S, Weimar C, Erbel R, Jöckel KH, et al. Associations between self-reported sleep characteristics and incident mild cognitive impairment: The Heinz Nixdorf Recall Cohort Study. Sc Rep. 2020;10:6542.

11. OM Bubu M Brannick J Mortimer O Umasabor-Bubu YV Sebastião Y Wen et al 2017 Sleep, Cognitive impairment, and Alzheimer's disease: A Systematic Review and Meta-Analysis Sleep 40

12. Xie L, Kang H, Xu Q, Chen MJ, Liao Y, Thiyagarajan M, et al. Sleep drives metabolite clearance from the adult brain. Science. 2013:342:373-7.

13. Ma XQ, Jiang $C Q$, Xu L, Zhang WS, Zhu F, Jin $Y L$, et al. Sleep quality and cognitive impairment in older Chinese: Guangzhou Biobank Cohort Study. Age Ageing. 2019:49:119-24.

14. Blackwell T, Yaffe K, Ancoli-Israel S, Redline S, Ensrud KE, Stefanick ML, et al. Osteoporotic Fractures in Men (MrOS) Study Group. Association of sleep characteristics and cognition in older community-dwelling men: the MrOS sleep study. Sleep. 2011:34:1347-56.

15. Leng M, Yin H, Zhang P, Jia Y, Hu M, Li G, et al. Sleep Quality and HealthRelated Quality of Life in Older People With Subjective Cognitive Decline, Mild Cognitive Impairment, and Alzheimer Disease. J Nerv Ment Dis. 2020;208:913.

16. Dlugaj M, Weinreich G, Weimar C, Stang A, Dragano N, Wessendorf TE, et al. Heinz Nixdorf Recall Study Investigative Group. Sleep-disordered breathing, sleep quality, and mild cognitive impairment in the genera population. J Alzheimers Dis. 2014;41:479-97.

17. Matsumoto T, Chin K. Prevalence of sleep disturbances: Sleep disordered breathing, short sleep duration, and non-restorative sleep. Respir Investig. 2019:57:227-37.

18. Lévy P, Kohler M, McNicholas WT, et al. Obstructive sleep apnoea syndrome. Nat Rev Dis Primers. 2015;1:15015.
19. Bubu OM, Andrade AG, Umasabor-Bubu OQ, et al. Obstructive sleep apnea, cognition and Alzheimer's disease: A systematic review integrating three decades of multidisciplinary research. Sleep Med Rev. 2020;50: 101250. https://doi.org/10.1016/j.smrv.2019.101250.

20. Leng Y, McEvoy CT, Allen IE, Yaffe K. Association of Sleep-Disordered Breathing With Cognitive Function and Risk of Cognitive Impairment: A Systematic Review and Meta-analysis. JAMA Neurol. 2017:74:1237-45.

21. Landry GJ, Liu-Ambrose T. Buying time: a rationale for examining the use of circadian rhythm and sleep interventions to delay progression of mild cognitive impairment to Alzheimer's disease. Front Aging Neurosci. 2014;6:325

22. Lo K, Woo B, Wong M, Tam W. Subjective sleep quality, blood pressure, and hypertension: a meta-analysis. J Clin Hypertens (Greenwich). 2018:20:592-605

23. Marti-Soler H, Hirotsu C, Marques-Vidal P, Vollenweider P, Waeber G, Preisig $\mathrm{M}$, et al. The NoSAS score for screening of sleep-disordered breathing: a derivation and validation study. Lancet Respir Med. 2016;4:742-8.

24. Hong C, Chen R, Qing S, Kuang A, Yang H, Su X, et al. Validation of the NoSAS Score for the Screening of Sleep-Disordered Breathing: A HospitalBased Retrospective Study in China. J Clin Sleep Med. 2018:14:191-7.

25. Rong Y, Wang S, Wang H, et al. Validation of the NoSAS Score for the Screening of Sleep-Disordered Breathing in a Sleep Clinic. Can Respir J. 2020;2020:4936423.

26. L Wang N Li M Heizhati X Yao G Duiyimuhan K Zhou et al 2019 Prevalence, Awareness, Treatment, and Control and Related Factors of Hypertension in Multiethnic Agriculture, Stock-Raising, and Urban Xinjiang, Northwest China: A Cross-Sectional Screening for 47000 Adults Int J Hypertens 3576853

27. Pan F, Heizhati M, Wang L, Zhou L, Hong J, Zhang D, et al. Distribution characteristics of circulating homocysteine and folate and related factors in agriculture, stock-raising and urban populations: a cross-sectional survey. Public Health Nutr. 2020;2:1-8.

28. Folstein MF, Folstein SE, McHugh PR, "Mini-mental state." A practical method for grading the cognitive state of patients for the clinician. J Psychiatr Res. 1975;12:189-98.

29. Wu L, He Y, Jiang B, Liu M, Wang J, Yang S, et al. The association between the prevalence, treatment and control of hypertension and the risk of mild cognitive impairment in an elderly urban population in China. Hypertens Res. 2016;39:367-75.

30. Buysse DJ, Reynolds CF 3rd, Monk TH, Berman SR, Kupfer DJ. The Pittsburgh Sleep Quality Index: a new instrument for psychiatric practice and research. Psychiatry Res. 1989;28:193-213.

31. Mollayeva T, Thurairajah P, Burton K, Mollayeva S, Shapiro CM, Colantonio A. The Pittsburgh sleep quality index as a screening tool for sleep dysfunction in clinical and non-clinical samples: a systematic review and meta-analysis. Sleep Med Rev. 2016;25:52-73.

32. Joint Committee for Guideline Revision. 2018 Chinese Guidelines for Prevention and Treatment of Hypertension-A report of the Revision Committee of Chinese Guidelines for Prevention and Treatment of Hypertension. J Geriatr Cardiol. 2019;16:182-241.

33. Alberti KG, Zimmet PZ. Definition, diagnosis and classification of diabetes mellitus and its complications. Part 1: diagnosis and classification of diabetes mellitus provisional report of a WHO consultation. Diabet Med. 1998;15:539-53.

34. Joint Committee for the Prevention and Treatment of Dyslipidemia in Chinese Adults 2016 Guidelines for the prevention and treatment of dyslipidemia in Chinese adults (2016 Revised Edition) Chin J Cardiol 44 833850

35. Cai J, Wu M, Ren J, Du Y, Long Z, Li G, et al. Evaluation of the Efficiency of the Reticulocyte Hemoglobin Content on Diagnosis for Iron Deficiency Anemia in Chinese Adults. Nutrients. 2017;9:450.

36. Zung WWK. How normal is anxiety? 1980.

37. Zung WW. From art to science. The diagnosis and treatment of depression. Arch Gen Psychiatry. 1973:29:328-37.

38. Mengesha MM, Roba HS, Ayele BH, Beyene AS. Level of physical activity among urban adults and the socio-demographic correlates: a population-based cross-sectional study using the global physical activity questionnaire. BMC Public Health. 2019;19:1160.

39. Kim JH. Multicollinearity and misleading statistical results. Korean J Anesthesiol. 2019:72:558-69. 
40. Kylstra WA, Aaronson JA, Hofman WF, Schmand BA. Neuropsychological functioning after CPAP treatment in obstructive sleep apnea: a metaanalysis. Sleep Med Rev. 2013;17:341-7.

41. Loredo JS, Ancoli-Israel S, Kim EJ, Lim WJ, Dimsdale JE. Effect of continuous positive airway pressure versus supplemental oxygen on sleep quality in obstructive sleep apnea: a placebo-CPAP-controlled study. Sleep. 2006;29:564-71.

42. Miller Ma, Wright H, Ji C, Cappuccio FP. Cross-sectional study of sleep quantity and quality and amnestic and non-amnestic cognitive function in an ageing population: the English Longitudinal Study of Ageing (ELSA). PLoS One. 2014;9:e100991.

43. Senaratna CV, Perret JL, Lodge CJ, Lowe AJ, Campbell BE, Matheson MC, et al. Prevalence of obstructive sleep apnea in the general population: A systematic review. Sleep Med Rev. 2017;34:70-81.

44. Aunjitsakul W, Pitanupong J, Werachattawan N. Sleep Quality among Elderly People in Songkhla Province, Thailand: A Two-Stage Cluster Sampling Study[J]. Journal of the Medical Association of Thailand = Chotmaihet thangphaet. 2018;101:137-44.

45. Fietze I, Laharnar N, Obst A, et al. Prevalence and association analysis of obstructive sleep apnea with gender and age differences - Results of SHIP-Trend. J Sleep Res. 2019;28:e12770.

46. Grandner MA, Martin JL, Patel NP, Jackson NJ, Gehrman PR, Pien G, et al. Age and sleep disturbances among American men and women: data from the U.S. Behavioral Risk Factor Surveillance System. Sleep. 2012;35:395-406.

47. Lara E, Koyanagi A, Olaya B, Lobo A, Miret M, Tyrovolas S, et al. Mild cognitive impairment in a Spanish representative sample: prevalence and associated factors. Int J Geriatr Psychiatry. 2016;31:858-67.

48. Zammit AR, Katz MJ, Zimmerman ME, Bitzer M, Lipton RB. Low eGFR is associated with dysexecutive and amnestic mild cognitive impairment. Alzheimers Dement (Amst). 2015:1:152-9.

49. Zhang MY, Katzman R, Salmon D, Jin H, Cai GJ, Wang ZY, et al. The prevalence of dementia and Alzheimer's disease in Shanghai, China: impact of age, gender, and education. Ann Neurol. 1990;27:428-37.

50. Pan H, Huang X, Li F, Ren M, Zhang J, Xu M, et al. Association among plasma lactate, systemic inflammation, and mild cognitive impairment: a community-based study. Neurol Sci. 2019;40:1667-73.

\section{Publisher's Note}

Springer Nature remains neutral with regard to jurisdictional claims in published maps and institutional affiliations.

Ready to submit your research? Choose BMC and benefit from:

- fast, convenient online submission

- thorough peer review by experienced researchers in your field

- rapid publication on acceptance

- support for research data, including large and complex data types

- gold Open Access which fosters wider collaboration and increased citations

- maximum visibility for your research: over $100 \mathrm{M}$ website views per year

At BMC, research is always in progress.

Learn more biomedcentral.com/submissions 